\title{
Malignant fibrous histiocytoma in a patient presenting with urinary system symptoms
}

\author{
Mustafa Resorlu ${ }^{1 *}$, Ozan Karatag ${ }^{1}$, Fatma Uysal ${ }^{1}$, Muhsin Ozturk ${ }^{1}$ \\ ${ }^{1}$ Canakkale Onsekiz Mart University, Faculty of Medicine, Radiology, Canakkale, Turkey
}

\section{SUMMARY}

Study conducted at Canakkale Onsekiz Mart University, Faculty of Medicine, Department of Radiology, Canakkale, Turkey

Article received: 3/15/2017 Accepted for publication: 5/7/2017

*Correspondence:

Canakkale Onsekiz Mart University, Terzioglu Yerleskesi

Canakkale - Turkey Postal code: 17100

mustafaresorlu77@gmail.com

http://dx.doi.org/10.1590/1806-9282.63.11.950
Malignant fibrous histiocytoma is a rare tumor. It is most commonly seen in individuals between the fifth and seventh decades of life, in extremities, and less frequently in the retroperitoneum. Although its etiology is not clearly known, radiotherapy, chemical agents, previous history of surgery, trauma and fracture, and Hodgkin lymphoma have been blamed. Leiomyosarcoma, liposarcoma and rhabdomyosarcoma should be taken into account in differential diagnosis. It is seen on computed tomography as a mass lesion with irregular borders and density similar to that of the surrounding muscle tissue. Necrotic and hemorrhagic components in the mass are characterized as heterogeneous low density areas. Fluid-fluid levels can be detected by computed tomography and magnetic resonance imaging.

Keywords: histiocytoma, malignant fibrous, tomography, magnetic resonance imaging.

\section{INTRODUCTION}

Boundaries of the retroperitoneal space are the diaphragm at the top, the levator muscles at the bottom, the parietal peritoneum in anterior aspect, and the vertebral column and psoas muscles at the posterior side. Tumors located in the retroperitoneum might be originated from kidney, pancreas or fatty tissue. Leiomyosarcoma, liposarcoma, fibrosarcoma and malignant fibrous histiocytoma $(\mathrm{MFH})$ are among the retroperitoneal malignant mesenchymal tumors. ${ }^{1}$

$\mathrm{MFH}$, also named malignant fibrous xanthoma or pleomorphic fibrous histiocytoma, has been first reported by O'Brien and Stout in $1964 .^{2}$ It is an aggressive and high-grade sarcoma. The most frequent complaints are palpable mass, pressure symptoms on surrounding organs and abdominal pain. Prognosis of these rare tumors originating from connective tissue depends on tumor diameter, number of mitoses and degree of differentiation. ${ }^{3}$ We present the case of a patient referred to our hospital who has been diagnosed with MFH following surgery.

\section{Case report}

A 61 year-old female who had a history of hypertension and diabetes for 5 years presented to our hospital with abdominal distension and complaints related with the urinary system. Her medical history showed that she had undergone a surgical procedure associated with her uterus and ovaries and that had received no medical treatment after the surgery. Her laboratory tests revealed blood glucose, white blood cell and hemoglobin levels at $165 \mathrm{mg} / \mathrm{dL}, 15,500 / \mathrm{mm}^{3}$ and $9.1 \mathrm{~g} / \mathrm{dL}$, respectively. No abnormalities were detected in other results of routine complete blood count (CBC) or biochemistry test parameters. A mass lesion with lobulated contours and irregular borders measuring $12 \mathrm{~cm}$ in diameter between the kidney and the psoas muscle at the left side was detected by computed tomography $(\mathrm{CT})$ and magnetic resonance imaging (MRI).

CT scan revealed a calcific component measuring $1 \mathrm{~cm}$ in diameter at the posteromedial side of the lesion (Figure 1). On MRI, the lesion was isodense on T1 weighted series and slightly hyperdense compared to the muscle on T2 weighted series. Focal areas with low signal indicating fibrous tissue were present in the lesion on $\mathrm{T} 2$ weighted series. Intense enhancement was seen in the series after contrast injection (Figure 2). The mass lesion, evaluated as a retroperitoneal sarcoma radiologically, was diagnosed as a malignant fibrous histiocytoma after histopathological examination following surgery. 

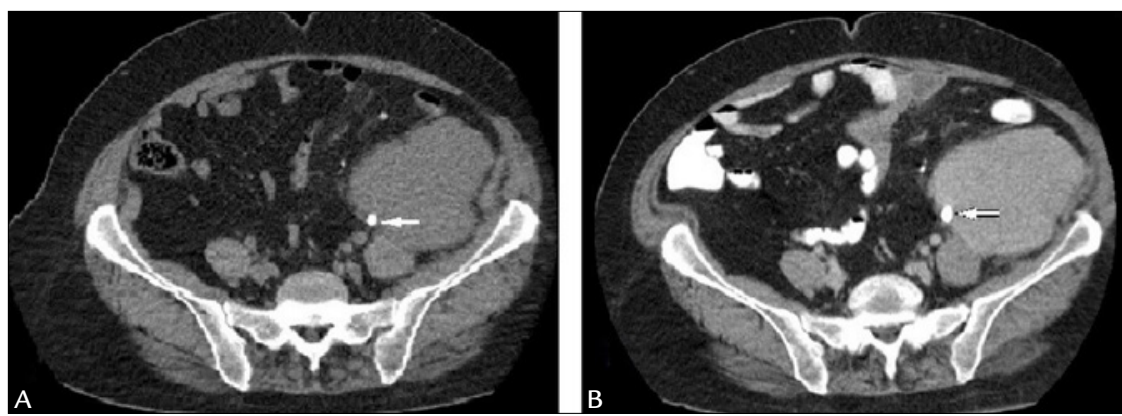

FIGURE 1 A. A mass lesion including a calcific component in close proximity with the psoas muscle in axial pre- (B) and post-contrast CT images.
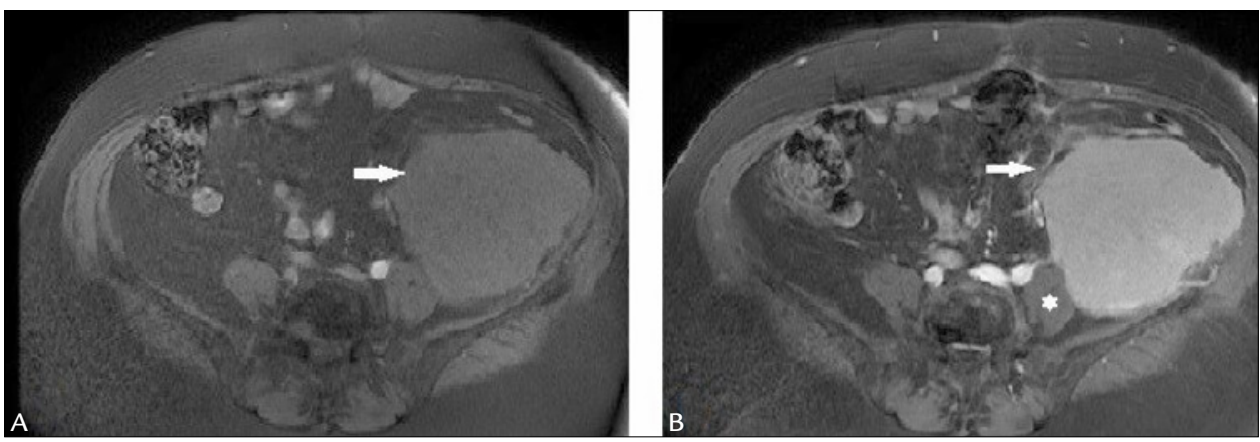

FIGURE 2 A. Intense contrast enhancement is observed in fat-suppressed T1-weighted pre- (B) and post-contrast MRI series.

\section{Discussion}

MFH originates from undifferentiated mesenchymal cells and comprises approximately $0.5 \%$ of all malignant tumors. ${ }^{4}$ They can occur at any age but are more frequent in relatively older individuals, especially in the fifth and seventh decades of life. They are most commonly seen in the extremities but can occur at any localization in the body. ${ }^{3}$ Malignant fibrous histiocytoma, which is one of the mesenchymal tumors, includes fibroblasts at different stages and histiocyte-like cells. They are divided into five different subgroups and the ones more commonly seen are pleomorphic and myxoid types, which tend to present higher grades, while the rarer giant cell, inflammatory and angiomatoid subtypes tend to present lower grades. ${ }^{5}$

The etiology of MFH is not completely known; however, it is more common in patients who received radiotherapy, similar to other sarcomas. In addition, sunlight, chemical agents such as phenacetin, previous surgery, trauma and fracture, bone infarct, and tumors such as Hodgkin lymphoma and multiple melanoma are predisposing factors. ${ }^{4,5}$ Most of the patients are asymptomatic, since the lesion is located retroperitoneally and deeply, or they have nonspecific symptoms such as flank pain, fever and weight loss. C-reactive protein level and erythrocyte sedimentation rate may be found to be increased in labo- ratory tests. ${ }^{6}$ Palpable mass, compression of surrounding organs and flank pain are the results of the increasing dimensions of the tumor. Also, cases of paraneoplastic syndrome have been reported in the literature. ${ }^{7}$

Differential diagnosis includes leiomyosarcoma, liposarcoma and rhabdomyosarcoma as retroperitoneal tumors. Psoas abscess, hematoma and hydatid cyst should also be considered in differential diagnosis. ${ }^{6}$ Radiological findings are nonspecific and the diagnosis is made histopathologically. The dimensions and site of the lesion, presence of hemorrhagic or necrotic components, and mass affecting the surrounding organs might be detected by radiological imaging. Ultrasonographically, the tumor has a hypoechoic mass character and necrotic/hemorrhagic components are seen as hypoechoic or anechoic images with septated formations. ${ }^{6,8}$ Typical CT findings include a large infiltrative mass with ill-defined borders and density similar to that of muscle tissue. Hypodense areas secondary to necrotic and hemorrhagic components and hyperdense calcific components cause non-homogeneity. Heterogeneous contrast enhancement might be seen in post-contrast images secondary to necrotic and hemorrhagic components. ${ }^{5}$ Fluid-fluid levels may be seen secondary to hemorrhage in CT and MRI. Signal properties of hemorrhagic component in MRI vary according 
to the character of the blood products. In addition, fibrous components demonstrate low signal on $\mathrm{T} 1$ and $\mathrm{T} 2$ weighted sequences. Although radiological findings are nonspecific, some specifications are present and can differentiate MFH from renal carcinoma. Large dimensions at the time of diagnosis, absence of renal vein or vena cava involvement, characteristic signs of fibrous components and necrotic/hemorrhagic or calcific components raise suspicion for MFH. ${ }^{9}$

The prognosis of MFH is generally poor. Determinant prognostic factors are subgroup type and location of the tumor, as well as presence of metastases or paraneoplastic syndrome. Five-year survival rate is $66 \%$ when tumor dimension is less than $5 \mathrm{~cm}$, decreasing to $30 \%$ if the diameter is greater than $5 \mathrm{~cm} .{ }^{3,10}$ Similarly, there is a positive correlation between tumor diameter and rate of metastasis. Prognosis is better in tumors located in extremities compared to retroperitoneal or deeply located tumors., ${ }^{9,10}$

Primary treatment of MFH is surgical excision of the mass. Radiotherapy plays an important role in the postsurgical treatment; however, the efficacy of chemotherapy is controversial. ${ }^{10}$

\section{References}

1. Gemici K, Buldu İ, Acar T, Alptekin H, Kaynar M, Tekinarslan E, et al. Management of patients with retroperitoneal tumors and a review of the literature. World J Surg Oncol. 2015; 13:143.

2. O'Brien JE, Stout AP. Malignant fibrous xanthomas. Cancer. 1964; 17:1445-55.

3. Hsu HC, Huang EY, Wang CJ. Treatment results and prognostic factors in patients with malignant fibrous histiocytoma. Acta Oncol. 2004; 43(6):530-5.

4. Gutierrez JC, Perez EA, Franceschi D, Moffat FL Jr, Livingstone AS, Koniaris LG. Outcomes for soft-tissue sarcoma in 8249 cases from a large state cancer registry. J Surg Res. 2007; 141(1):105-14.

5. Karki B, Xu YK, Wu YK, Zhang WW. Primary malignant fibrous histiocytoma of the abdominal cavity: CT findings and pathological correlation. World J Radiol. 2012; 4(4):151-8.

6. Gupta MM, Bahri N, Watal P, Rathod K, Thaker S, Bhandari P, et al. Retroperitoneal cystic malignant fibrous histiocytoma mimicking a psoas abscess. Iran J Radiol. 2015; 12(3):e17507.

7. Kageyama K, Moriyama T, Hizuka N, Sakihara S, Takayasu S, Tamasawa N, et al. Hypoglycemia associated with big insulin-like growth factor II produced during development of malignant fibrous histiocytoma. Endocr J. 2003; 50(6):753-8

8. Rajiah P, Sinha R, Cuevas C, Dubinsky TJ, Bush WH Jr, Kolokythas O. Imaging of uncommon retroperitoneal masses. Radiographics. 2011; 31(4):949-76

9. Nishino M, Hayakawa K, Minami M, Yamamoto A, Ueda H, Takasu K. Primary retroperitoneal neoplasms: CT and MR imaging findings with anatomic and pathologic diagnostic clues. Radiographics. 2003; 23(1):45-57.

10. Kearney MM, Soule EH, Ivins JC. Malignant fibrous histiocytoma: a retrospective study of 167 cases. Cancer. 1980; 45(1):167-78. 